\title{
Comment on: "Interseismic Strain Accumulation near Lisbon (Portugal) from Space Geodesy" by Fonseca et al. (2021)
}

\author{
Luis Matias ${ }^{\mathrm{a}}$ \\ a Instituto Dom Luiz, Faculdade de Ciências da Universidade de Lisboa, 1749-016 Lisboa, \\ Portugal,Imatias@fc.ul.pt
}

This manuscript, as a comment to one article that appeared in 2021 in Geophysical Research Letters (GRL), will not be subject to peer review since it is the policy of GRL not to publish comments and replies, https://www.agu.org/Publish-with-AGU/Publish/\#1. The only procedure found to open a discussion on the subject was the publication in EarthArxive. Please contact the author with any questions or concerns, or use the EarthArxive comment forum. 


\title{
Comment on: "Interseismic Strain Accumulation near Lisbon (Portugal) from Space Geodesy" by Fonseca et al. (2021)
}

\author{
Luis Matias ${ }^{\mathrm{a}}$ \\ ${ }^{a}$ Instituto Dom Luiz, Faculdade de Ciências da Universidade de Lisboa, 1749-016 Lisboa, \\ Portugal
}

\section{Summary}

The paper by Fonseca et al. (2021), hereafter referred as FON21, published in Geophysical Research Letters ${ }^{2}$ make several conclusions that are not convincingly supported by the evidence of the data that is made available. In this comment we will address the following statements: 1) FON21 "provides new evidence of sinistral simple shear driven by a NNESSW first-order tectonic lineament; 2) "PSInSAR vertical velocities corroborate qualitatively the GNSS strain-rate field, showing uplift/subsidence where the GNSS data indicate contraction/extension"; 3) FON21 proposes "the presence of a small block to the W of Lisbon moving independently toward the SW with a relative velocity of $0.96 \pm 0.20$

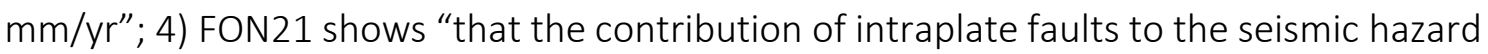
in the LMA is more important than currently assumed". We conclude that more evidence needs to be collected to confirm or infirm FON21 statements and conclusions. For the moment the proposal of an autonomous crustal block moving with significant velocity in relation to the neighboring domain should be considered speculative and unproved.

\section{The Lower Tagus Valley seismotectonics and seismic hazard}

The Lisbon Metropolitan Area (LMA) has suffered in the past from strong ground motions generated by earthquakes in the surrounding geographical area which is generally designated as the Lower Tagus Valley (LTV). Of these, two deserve special relevance, the $26^{\text {th }}$ January 1531 and the $23^{\text {rd }}$ April 1909 events. Several authors determined the magnitude of the 1909 event as Mw 6.0-6.2 (Teves-Costa et al., 1999; Dineva et al., 2002; Stich et al., 2005, Teves-Costa and Batlló, 2011). The 2011 value (6.0) was the one retained for the SHEEC Catalogue (1900-2006), Grünthal et al. (2013). Baptista et al. (2014) revised the macroseismic field for the 1531 earthquake and proposed $\mathrm{Mw}=6.34$ \pm 0.17 . The SHEEC Catalogue (1000-1899), Stucchi et al. (2013) proposes a moment magnitude $\mathrm{Mw}=6.47 \pm 0.49$. These values are much more conservative than the ones presented by FON21, 7 for the 1531 event and 6.0 to 6.5 for the 1969 one.

Earthquake recurrence models and maximum magnitude estimates have been used by several authors to estimate the probabilistic seismic hazard affecting the LMA. Given the poor knowledge of active faults, these studies prefer the areal source model and consider the LTV as one zone. Uncertainties in earthquake recurrence model parameters and

\footnotetext{
${ }^{1}$ Open access at: https://agupubs.onlinelibrary.wiley.com/doi/full/10.1029/2021GL096862

${ }^{2}$ Comments and replies are not allowed in Geophysical Research Letters, https://www.agu.org/Publishwith-AGU/Publish/\#1
} 
differences on the choice of the best ground motion prediction equations let to the publication of PSHA results that differ strongly (e.g. Vilanova and Fonseca, 2007, Campos Costa et al., 2008, Mezcua et al., 2011, Woessner et al., 2015). These discrepancies may lead to confusion among the general public and policy makers and may lead to difficulties in the acceptance of building codes that are established from one of the studies.

In the following sections we will concentrate our analysis on the restricted area defined in FON21 figures $1 \mathrm{a}$ and $2 \mathrm{a}$.

\section{The rigid block identified by FON21}

Investigating the GNSS derived velocities with respect to Eurasia, FON21 identified in figure 2a three locations, MAFR, PACO and IGPO, that moved with a velocity of $0.96 \pm 0.20$ $\mathrm{mm} / \mathrm{yr}$ relative to the other 8 locations in the area. To reach this value FON21 discarded the CASC location (close to PACO) arguing that CASC is "pinned by the Sintra batholith that lays to the North. Later, FON21 present in figure $4 b$ the tectonic model that could explain the block kinematics.

We begin the discussion by presenting in Fig. 1 the kinematic data relative to the FON21 reference block.

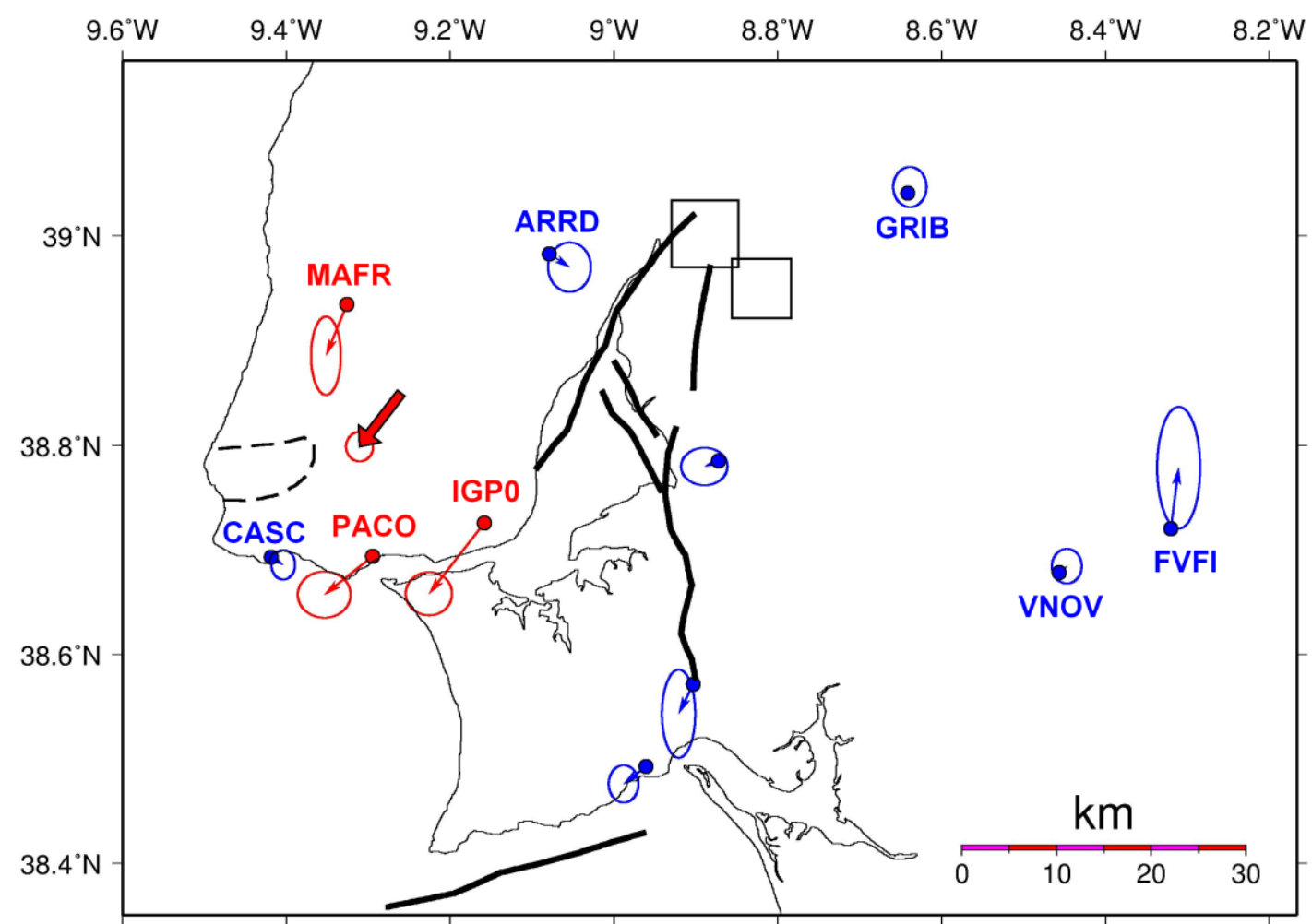

Fig. 1-GNSS velocities relative to the rigid block formed by the stations in blue. The red arrows show the velocities of the sites included in the rigid block defined by FON21. The

thick red arrow shows the average velocity between the red and blue sites. The rectangles show the location of the 1531 and 1909 earthquakes mentioned in the text. Thick lines show the active tectonics model used by FON21 for the interpretation of the GNSS velocities. The small dashes show the location of the Sintra batholith. 
The southern and eastern rigid block limits are not provided in FON21 but they can be inferred from the GNSS sites. The southern limit lies probably along the E-W Tagus river or south of it. The eastern boundary must lie somewhere between the MAFR and ARRD sites. None of these presumed boundaries is explained by the FON21 tectonic model. If indeed the LTV faults represent a major tectonic feature, then site ARRD should move differently from the other reference sites and should move with a velocity close to the rigid block sites. The interpretation of the ARRD velocity in the light of the tectonic model proposed is not given in FON21.

If CASC is pinned to the Sintra batholith and it moves with the same velocity as the other reference sites, it means that CASC is probably moving with the basement velocity. Then the meaning and relevance of the velocities derived for MAFR, PACO and IGPO may be questioned. Do they represent the velocity of a rigid block with crustal thickness or do they show a shallow feature that might be detached from the basement, as questioned by FON21? In this area the crustal thickness is $26 \pm 2 \mathrm{~km}$ (Dündar et al., 2016), approximately the length between the three sites that define the FON21 rigid block. As mentioned by FON21 the rigid block may extend further West, but no evidence is provided for that. If the rigid block proposed has crustal expression, then the aspect ratio is close to $1 \times 1 \times 1$, which may be considered small and difficult to assess with sparse data.

We may also question the interpretation of CASC velocity in the light of the seismicity observed in the area and presented in FON21 figure 1b. If the Sintra batholith is resisting the block movement then a higher seismicity rate should be expected surrounding it, which is not observed.

\section{The rigid block velocity}

FON21 associated three GNSS sites to the rigid block and discarded one site, very close to this set. Since we disputed in the previous section the reasons for discarding CASC, the procedure used by FON21 might be considered cherry picking. In this section we consider all possible sets of 3 close GNSS sites, without cherry picking, and compare their average velocity with the average velocity of the other 8 sites that are considered as reference. The results are presented in Table 1 and compared the rigid block proposed in FON21.

We may conclude that, from the 9 possible combination of 3 close GNSS sites without cherry picking, 5 combinations show a significant velocity relative to the other 8 sites considered as reference, higher than $0.5 \mathrm{~mm} / \mathrm{yr}$. Using the same reasoning of FON21 one could argue that FVFI, VNOV and GRIB could define a rigid block with a relative velocity of $0.84 \pm 0.07 \mathrm{~mm} / \mathrm{yr}$. Our conclusion is that the definition of the rigid block made by FON21 is somewhat arbitrary, guided by model considerations and not driven by the data. We may also infer that, if there is one rigid block in the LTV area, then its relative motion must stand out from the more or less random choice of sites. Then, the relative velocity attributed by FON21 for the proposed rigid block is clearly over-estimated. 


\begin{tabular}{|c|c|c|}
\hline \multicolumn{3}{|c|}{$\begin{array}{r}\text { Table 1 - Relative velocity between the } 3 \text { closer sites } \\
\text { and the other } 8 \text { considered as reference }\end{array}$} \\
\hline & V (mm/yr) & Azimuth \\
\hline MAFR PACO IGPO & $0.96 \pm 0.07$ & $218 \pm 5$ \\
\hline CASC PACO IGPO & $0.61 \pm 0.08$ & $218 \pm 8$ \\
\hline MAFR ARRD PACO & $0.40 \pm 0.03$ & $194 \pm 11$ \\
\hline ARRD MAFR ALCX & $0.21 \pm 0.07$ & $143 \pm 21$ \\
\hline ARRA PALM IGPO & $0.62 \pm 0.07$ & $218 \pm 7$ \\
\hline PALM ARRA ALCX & $0.12 \pm 0.07$ & $242 \pm 38$ \\
\hline ALCX PALM IGPO & $0.52 \pm 0.07$ & $219 \pm 9$ \\
\hline GRIB ALCX ARRD & $0.35 \pm 0.07$ & $54 \pm 14$ \\
\hline VNOV FVFI ALCX & $0.73 \pm 0.07$ & $19 \pm 6$ \\
\hline FVFI VNOV GRIB & $0.84 \pm 0.07$ & $24 \pm 5$ \\
\hline
\end{tabular}

\section{Testing the LTV alignment with GNSS}

We may expand the discussion provided in the previous section by assessing the relevance of the LTV alignment to explain the observed GNSS velocities. For this we consider the 11l GNSS sites separated into Western and Eastern blocks, as shown in Fig. 2.

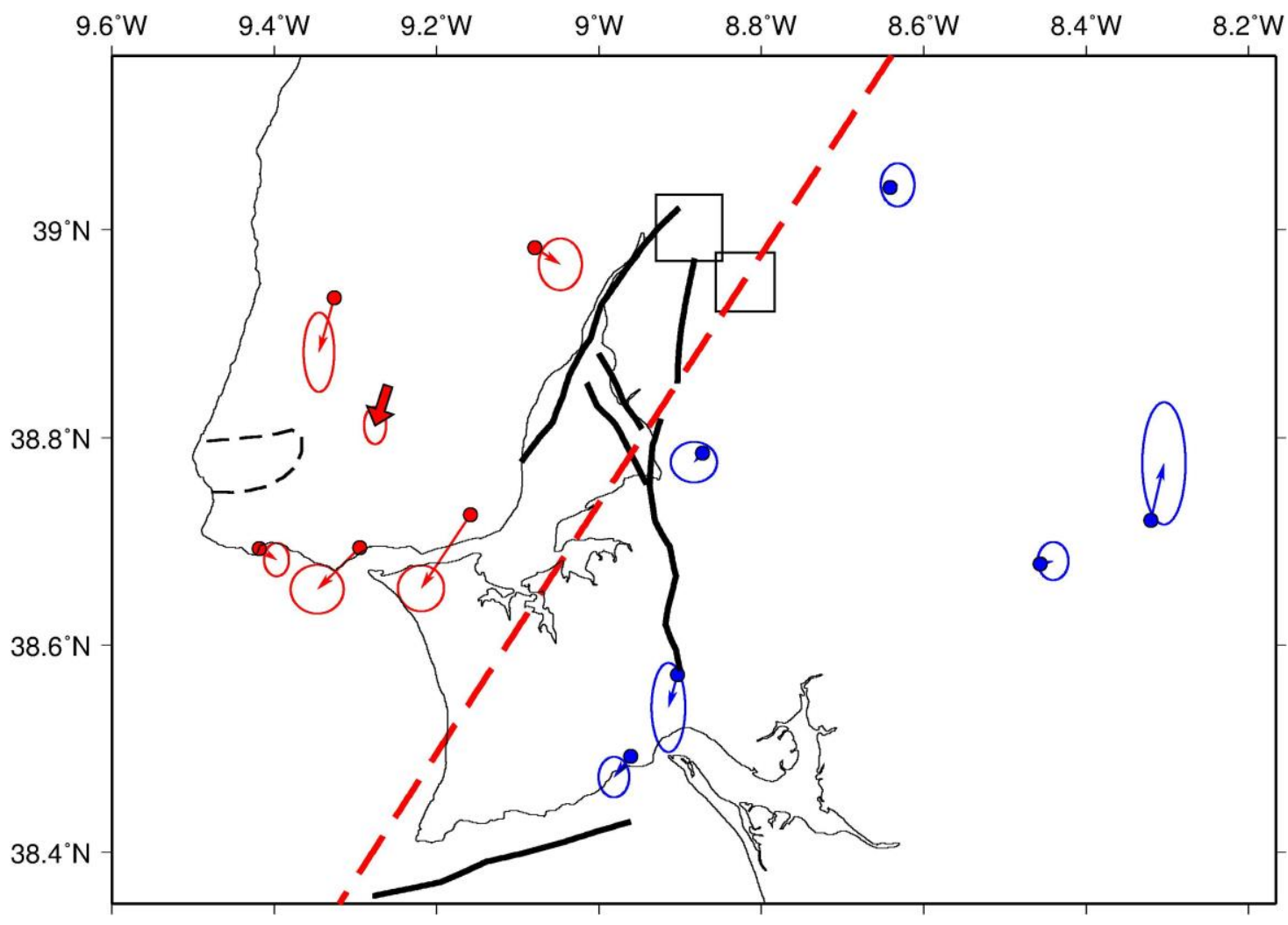

Fig. 2 - GNSS velocities relative to the rigid block formed by the stations in blue, east of the LTV alignment (dashed red line). The red arrows show the velocities of the sites West of the LTV alignment. The thick red arrow shows the average velocity between the red and blue sites. Other features as in Fig. 1. 
The Western side of the LTV alignment moves relative to the Eastern side with an average velocity of $0.60 \pm 0.04$ and an azimuth $198 \pm 7$. This velocity is smaller than the relative velocity of the rigid block defined by FON21 and may be considered only marginally relevant in the light of the considerations made in the previous section.

Looking into the GNSS velocities, we may test if a fictitious NW-SE tectonic alignment may also explain the observations (Fig. 3).

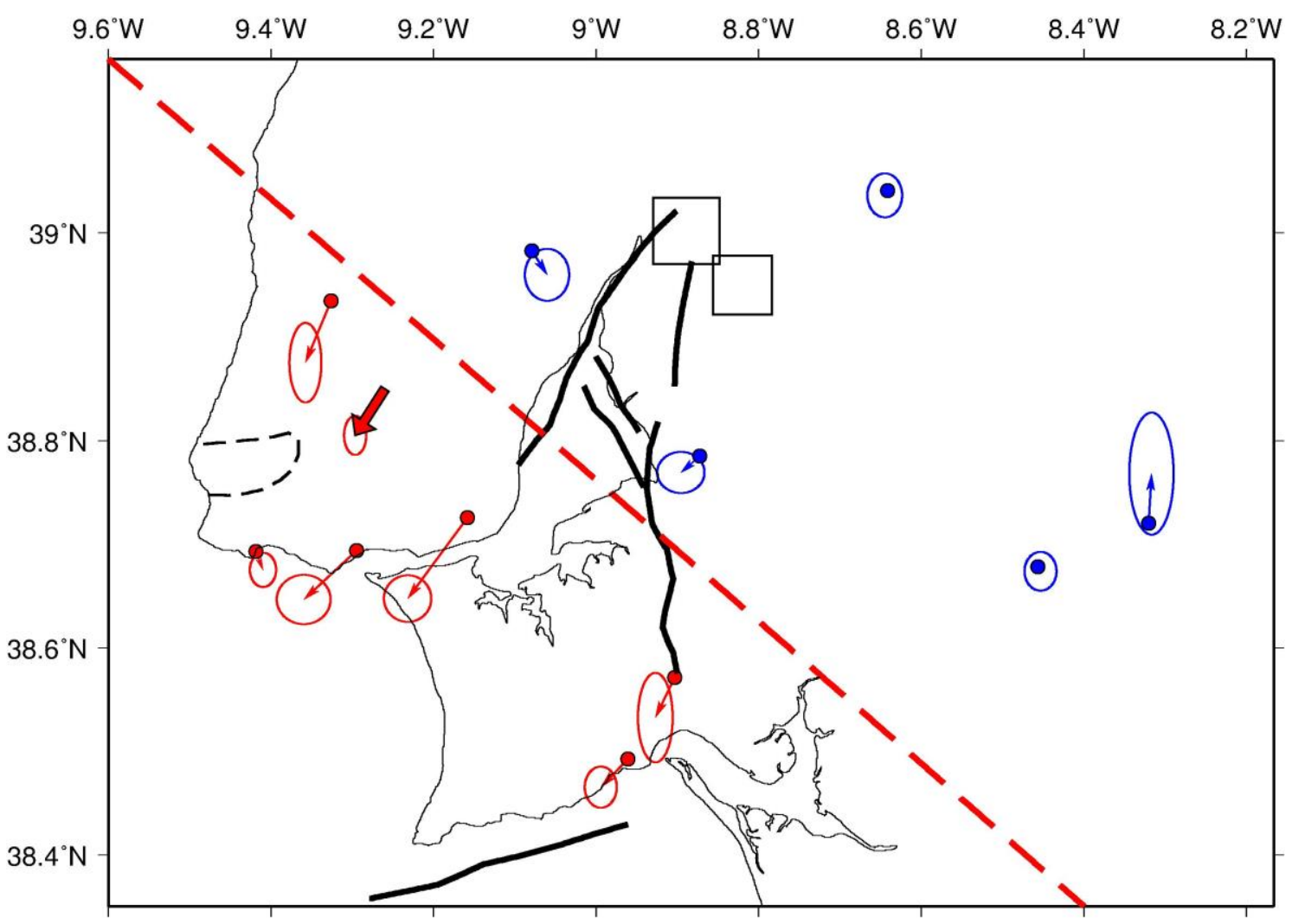

Fig. 3-GNSS velocities relative to the rigid block formed by the stations in blue, north of a fictitious alignment (dashed red line). The red arrows show the velocities of the sites south of the alignment. The thick red arrow shows the average velocity between the red and blue sites. Other features as in Fig. 1.

In this fictitious example, the sites SW of the alignment move relative to the NE side with an average velocity of $0.79 \pm 0.07$ and an azimuth $212 \pm 6$. This is a more relevant velocity than the one obtained with the LTV alignment, without cherry picking. Our conclusion is that the available GNSS data is not adequate to discuss tectonic models that are elaborated to explain the seismicity and deformation in the LTV region.

\section{PSInSAR vertical velocities and the GNSS strain-rate field}

To facilitate the discussion of the GNSS strain-rate field we show in Fig. 4 a restricted version of FON21 figure with the same geographical limits as the ones given in previous figures of this comment. We also add some relevant features, the location of the GNSS sites identifying those that belong to the rigid block defined in FON21 and we add also the tectonic model used by FON21 for the interpretation. 


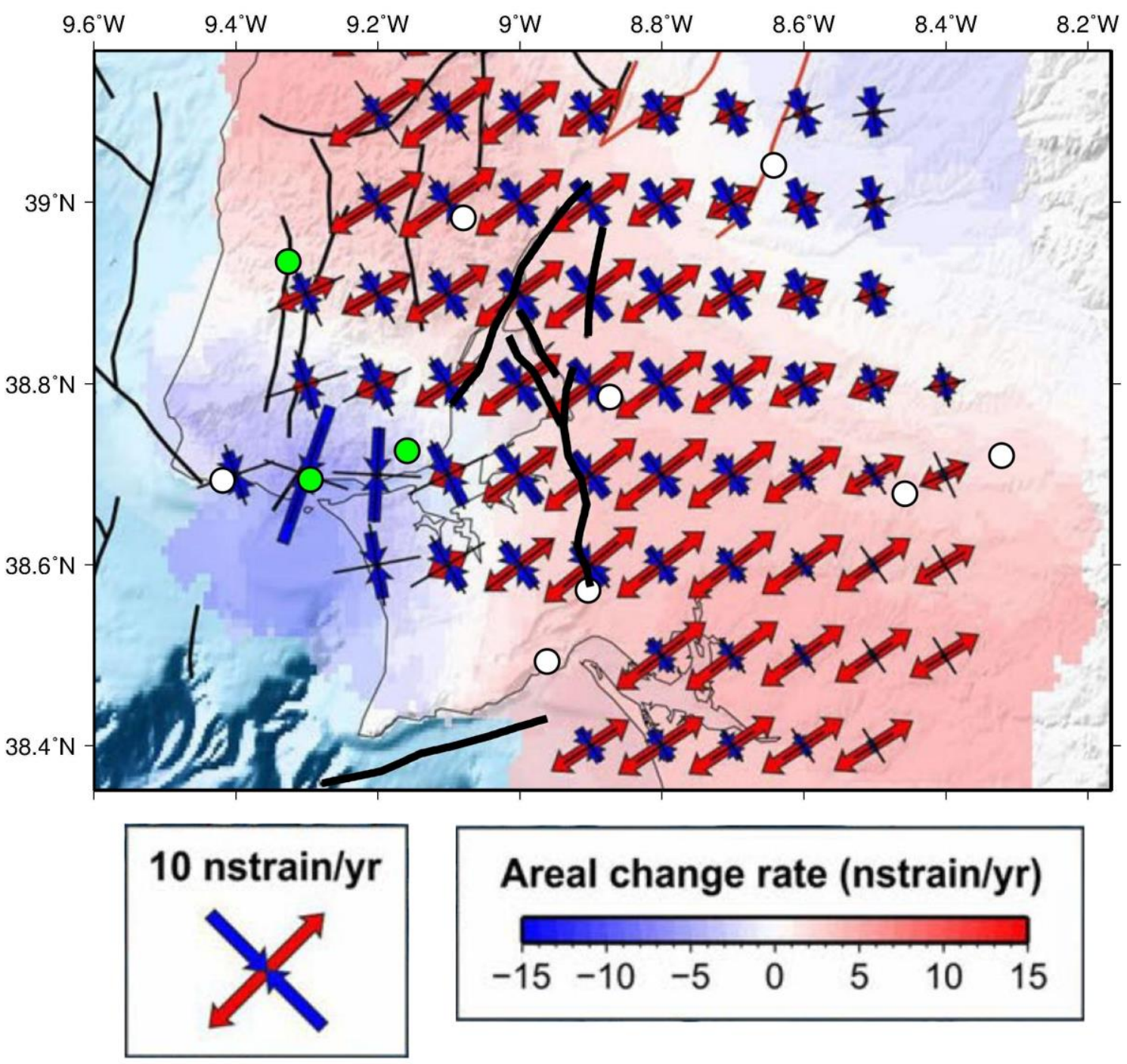

Fig. 4 - Strain-rates inferred from the GNSS horizontal velocities, modified from FON21. Blue corresponds to areas under compression, pink to areas under extension. Green circles show the GNSS sites used by FON21 to define a rigid block. The other GNSS sites are shown as white circles. Thick lines show the active tectonics model used by FON21 for the interpretation of the GNSS velocities

As mentioned in FON21 the whole domain where reliable strain-rates could be estimated is under extension, except for a small domain surrounding the Lisbon-Tagus bar area, North and South of the Tagus river. We may add that the LTV alignment or the tectonic model presented in FON21 do not show up on the strain-rate map. We remark also that the area where the rigid block proposed by FON21 is proposed in the area where the largest variation of strain-rate is found. Considering the 3 GNSS sites used by FON21, the strain regime changes $\mathrm{N}-\mathrm{S}$ from extension to compression and the maximum compression axis rotates from NNW-SSE to NNE-SSW in the North-South direction. These observations do not favor the proposal of FON21 that a rigid block exists west of the LTV alignment. 
As regards the PSInSAR vertical velocities we found the information presented in FON21 figure $3 a$ difficult to read. Using the data provided we preferred to compute a representative grid with the average vertical velocity on a cell size $\sim 1.1 \times 0.8 \mathrm{~km}^{2}(0.001$ 은 $x 0.001$ ) . We plot this grid on Fig. 5 with the same geographical limits used previously to facilitate comparing information.

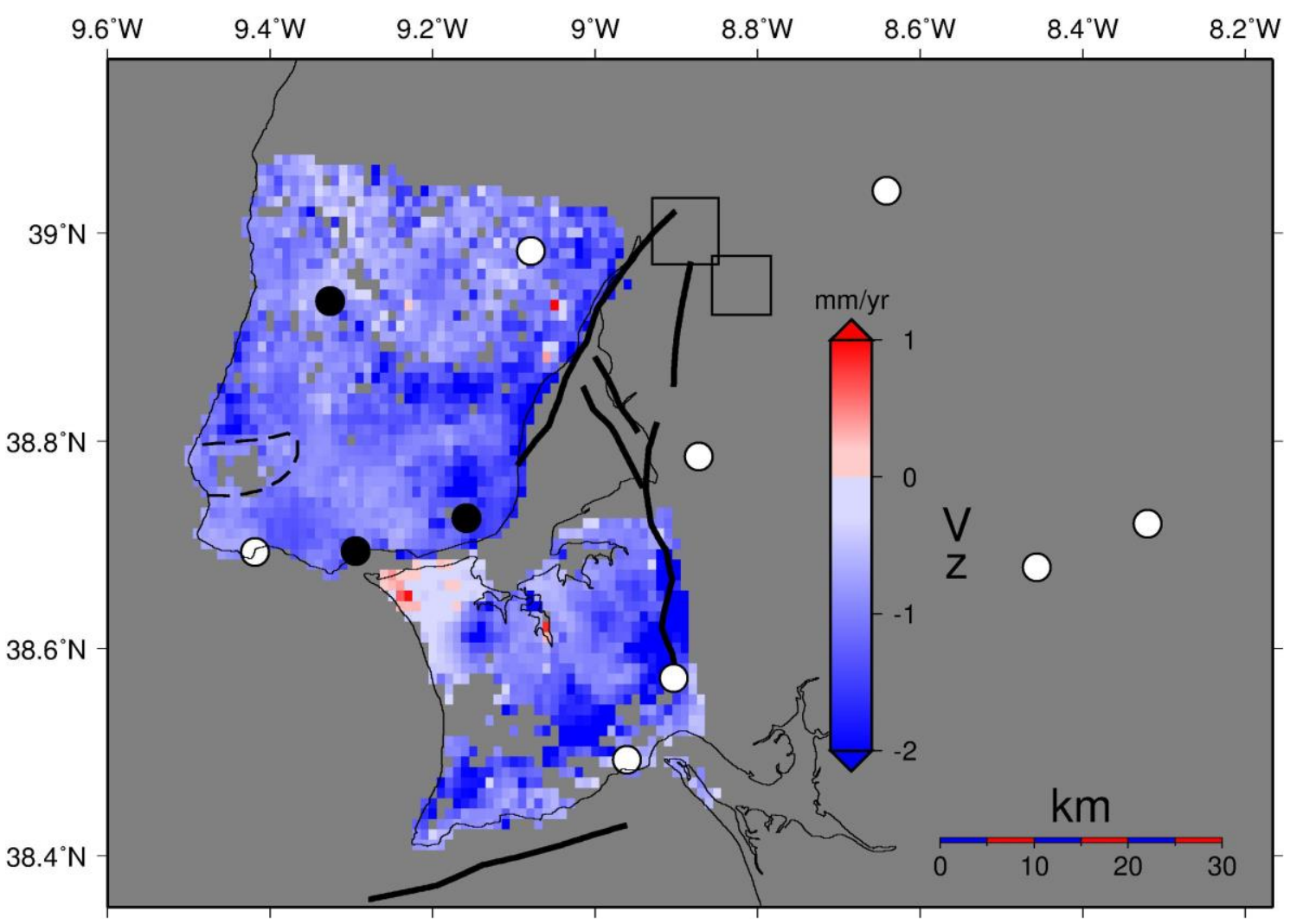

Fig. 5 - InSAR vertical velocities in the study area after averaging on a cell size $0.001^{\circ} \times 0.001 \%$. Data provided in FON21 where processing is explained. Positive velocities (shades of red) correspond to uplift, negative velocities (shades of blue) correspond to subsidence. Black circles show the GNSS sites used by FON21 to define a rigid block. The other GNSS sites are shown as white circles. Other features as in Fig. 1.

Our interpretation of the PSInSAR vertical velocities, based on Fig. 5, is very different from the one given in FON21. The whole domain is characterized by subsidence with different intensities. There is no correlation whatsoever with the rigid block proposed by FON21. Given its limited coverage to the East, the PSInSAR vertical velocities presented cannot confirm or infirm the tectonic model proposed by FON21 or the tectonic relevance of LTV alignment.

Comparing the strain compression and extension domains defined by GNSS (Fig. 4) with the vertical velocities in Fig. 5 we cannot agree with FON21 sentence that "PSInSAR vertical velocities corroborate qualitatively the GNSS strain-rate field, showing uplift/subsidence where the GNSS data indicate contraction/extension". In fact, significant uplift is restricted to very small domains, on the southern margin of the Tagus mouth. These localized points are surrounded by a near null subsidence, no compression 
here. The northern margin of the Tagus mouth, where compression is estimated by GNSS data, shows clear subsidence, in opposition to FON21 remark.

\section{Discussion on seismic hazard}

The estimated relative velocity of $0.96 \pm 0.20 \mathrm{~mm} / \mathrm{yr}$ of a crustal block made by FON21, when converted to seismic moment release, is compared to the seismic moment release proposed by Woessner et al. (2015) for the areal source area that includes the LTV. These authors considered for PSHA a logic tree with 3 possible maximum magnitudes for this source area of 7.1, 7.4 and 7.6. Using $15 \mathrm{~km}$ as the seismogenic thickness, FON21 concludes that the paper estimates agrees well with the recurrence model with 7.4 as the maximum magnitude, suggesting also a strong seismic coupling for the area.

We have shown on the previous sections that the definition of a rigid seismic block and its relative velocity is largely uncertain and most probably over-estimated. The maximum magnitude used by Woessner et al. (2015) on the earthquake recurrence models exceeds the maximum historical event of 1531 by one degree which also suggests that the moment magnitude release is over-estimated.

The seismic hazard affecting the LMA remains a challenge given the scarce evidence provided by the available data. In our opinion FON21 does not provide convincing evidence to comfort the earthquake recurrence model of Woessner et al. (2015).

\section{References}

Baptista, M. A., Miranda, J. M., \& Batlló, J. (2014). The 1531 Lisbon earthquake: A tsunami in the Tagus estuary?. Bulletin of the Seismological Society of America, 104(5), 2149-2161.

Costa, A. C., Sousa, M. L., \& Carvalho, A. (2008). Seismic zonation for Portuguese national annex of Eurocode 8. Proc. 14WCEE (Beijing, China, October 12-17).

Dineva, S., Batllo, J., Mihaylov, D., \& Van Eck, T. (2002). Source parameters of four strong earthquakes in Bulgaria and Portugal at the beginning of the 20th century. Journal of Seismology, 6(1), 99-123.

Dündar, S., Dias, N. A., Silveira, G., Kind, R., Vinnik, L., Matias, L., \& Bianchi, M. (2016). Estimation of the crustal bulk properties beneath mainland Portugal from P-wave teleseismic receiver functions. Pure and Applied Geophysics, 173(6), 1949-1970.

Fonseca, J. F., Palano, M., Falcão, A. P., Hrysiewicz, A., \& Fernandez, J. (2021). Interseismic Strain Accumulation near Lisbon (Portugal) from Space Geodesy. Geophysical Research Letters, e2021GL096862.

Grünthal, G., Wahlström, R., \& Stromeyer, D. (2013). The SHARE European Earthquake Catalogue (SHEEC) for the time period 1900-2006 and its comparison to the EuropeanMediterranean Earthquake Catalogue (EMEC). Journal of seismology, 17(4), 1339-1344.

Mezcua, J., Rueda, J., \& Blanco, R. M. G. (2011). A new probabilistic seismic hazard study of Spain. Natural hazards, 59(2), 1087-1108. 
Stich, D., Batlló, J., Macià, R., Teves-Costa, P., \& Morales, J. (2005). Moment tensor inversion with single-component historical seismograms: The 1909 Benavente (Portugal) and Lambesc (France) earthquakes. Geophysical Journal International, 162(3), 850-858.

Stucchi, M., Rovida, A., Capera, A. G., Alexandre, P., Camelbeeck, T., Demircioglu, M. B., ... \& Giardini, D. (2013). The SHARE European earthquake catalogue (SHEEC) 10001899. Journal of Seismology, 17(2), 523-544.

Teves-Costa, P., \& Batlló, J. (2011). The 23 April 1909 Benavente earthquake (Portugal): macroseismic field revision. Journal of Seismology, 15(1), 59-70.

Teves-Costa, P., Borges, J. F., Rio, I., Ribeiro, R., \& Marreiros, C. (1999). Source parameters of old earthquakes: semi-automatic digitization of analog records and seismic moment assessment. Natural Hazards, 19(2), 205-220.

Vilanova, S. P., \& Fonseca, J. F. (2007). Probabilistic seismic-hazard assessment for Portugal. Bulletin of the Seismological Society of America, 97(5), 1702-1717.

Woessner, J., Laurentiu, D., Giardini, D., Crowley, H., Cotton, F., Grünthal, G., ... \& Stucchi, M. (2015). The 2013 European seismic hazard model: key components and results. Bulletin of Earthquake Engineering, 13(12), 3553-3596. 